\title{
ON A CLASS OF EIGENVALUE PROBLEMS OF ORTHOTROPIC PLATES*
}

\author{
BY \\ CHIRUVAI P. VENDHAN AND SUBROTO KUMAR BHATTACHARYYA \\ Indian Institute of Technologv, Madras, India
}

\begin{abstract}
A class of eigenvalue problems for linear bending of semi-infinite orthotropic plates under the action of in-plane stress resultant in the finite direction has been developed. The structure of the eigenvalues has been investigated analytically for the case of simply supported plates and their physical basis underscored. It is shown that the treatment results in a coupled eigenvalue problem of bending as well as bifurcation buckling, thereby suggesting an alternate criterion for buckling.
\end{abstract}

1. Introduction. The elastostatic semi-infinite strip problem with homogeneous edge conditions and arbitrary end loading can be studied by the eigenfunction approach, wherein the eigenvalues denote the decay of the field quantities from the ends. Such a study provides a quantitative picture of the Saint Venant's principle. Fadle [1] and Papkovich [2,3] developed eigenfunctions for isotropic strips with in-plane stresses, which were later studied by many investigators [4]. A similar approach to the bending problem has been studied by Morley [5] and Gaydon [6] for isotropic plates and by Ramachandra Rao et al [7] for orthotropic plates. The emphasis in these works has been on the use of the eigenfunction expansion for the solution of finite plates. The generalized orthogonality relation due to Papkovich [2] may be used for determining the eigenseries expansion coefficients for certain boundary conditions. Archer and Bandyopadhyay [8] have studied the eigenfunctions in the context of thick plates based on the Reissner theory. The problem of bending of thin plates with in-plane forces is of considerable theoretical and practical interest. This paper considers the eigenfunction formulation of thin, elastic, semi-infinite orthotropic plates with in-plane stress in the finite direction only. Such a formulation integrates the eigenproblem of bending with that of linear bifurcation buckling. The structure of the eigenproblem has been investigated in detail for the case of a simply supported plate which is analytically tractable. The effect of the material

\footnotetext{
* Received November 1, 1984.
} 
parameters and the in-plane force is brought out by numerical examples. The integrated eigenproblem suggests an alternate mathematical criterion for buckling which merits further investigation in more general situations.

2. Solution of orthotropic plate equation. The linear bending of orthotropic plates with in-plane forces is governed by the well-known equation [9]:

$$
\begin{gathered}
D_{x} w_{, x x x x}+2\left(2 D_{x y}+D_{1}\right) w_{, x x y y}+D_{y} w_{, y y y y} \\
=a^{2}\left(N_{x} w_{, x x}+N_{y} w_{, y y}+2 N_{x y} w_{, x y}\right)
\end{gathered}
$$

where $w$ is the nondimensional transverse deflection $(w=\bar{w} / a)$ in the $(x, y)$ system shown in Fig. $1,($,$) denotes partial derivatives, D_{x}, D_{y}, D_{1}, D_{x y}$ are the various bending rigidities of the plate and the quantities $N_{x}, N_{y}$, and $N_{x y}$ are the in-plane stress resultants. Considering the in-plane force acting in the $x$-direction only, one has

$$
N_{y}=N_{x y}=0, \quad N_{x} \neq 0 .
$$

Thus (1) reduces to

$$
D_{x} w_{, x x x x}+2\left(2 D_{x y}+D_{1}\right) w_{, x x y y}+D_{y} w_{, y y y}-a^{2} N_{x} w_{, x x}=0 .
$$

The solution of (3) is sought in the form

$$
w(x, y)=X(x) e^{-\lambda y},
$$

using which, (3) reduces to an ordinary differential equation in $X$ :

$$
X^{\mathrm{iv}}+2\left(c_{1} \lambda^{2}-T\right) X^{\prime \prime}+c_{2} \lambda^{4} X=0
$$

where

$$
H=2 D_{x y}+D_{1}
$$

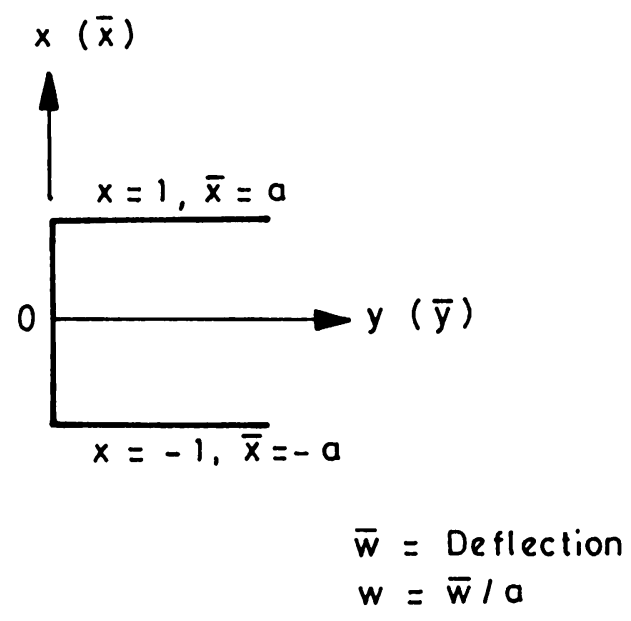

FIG. 1. Plate region $R$. 
and

$$
c_{1}=H / D_{x}, \quad c_{2}=D_{y} / D_{x}, \quad T=a^{2} N_{x} / D_{x} .
$$

The solution to (5) is sought in the form

$$
X(x)=e^{\alpha x}
$$

resulting in a characteristic equation

$$
\alpha^{4}+p \alpha^{2}+q=0
$$

where

$$
p=2 c_{1} \lambda^{2}-T, \quad q=c_{2} \lambda^{4}
$$

The roots of (9) are

$$
\alpha= \pm r, \pm s
$$

where

$$
r, s=\left\{\frac{-p \pm\left(p^{2}-4 q\right)^{1 / 2}}{2}\right\}^{1 / 2}
$$

The solution of (5) can therefore be written, for $r \neq s$, as

$$
X(x)=A \cosh r x+B \cosh s x
$$

or

$$
X(x)=A \sinh r x+B \sinh s x .
$$

The constants $A$ and $B$ can be evaluated using the edge conditions, where (13) holds good for the symmetric and (14) for the antisymmetric edge conditions, respectively.

For $r=s$, the solution of (5) can be written as

$$
X(x)=A \cosh r x+B x \sinh r x
$$

for the symmetric edge condition, and

$$
X(x)=A \sinh r x+B x \cosh r x
$$

for the antisymmetric edge condition. When the edge conditions are homogeneous, the above formulation leads to an eigenvalue problem which is investigated for different cases in the following sections.

3. Eigensolution. Consider a semi-infinite orthotropic plate in the region $R$ (see Fig. 1) given by

$$
R=\{-1 \leqslant x \leqslant 1,0 \leqslant y<\infty\} .
$$

For simply supported edges,

$$
w=w_{, x x}=0 \quad \text { at } x= \pm 1,
$$

which, in view of (4), yields

$$
X=X^{\prime \prime}=0 \quad \text { at } x= \pm 1
$$


Considering the symmetric solution (13), the use of the above results in

$$
\begin{aligned}
& A \cosh r+B \cosh s=0, \\
& A r^{2} \cosh r+B s^{2} \cosh s=0 .
\end{aligned}
$$

For the nontrivial solution of the above set of equations, the determinant of the associated coefficient matrix should vanish and hence

$$
\left(r^{2}-s^{2}\right) \cosh r \cosh s=0
$$

or

$$
F(\lambda, T)=\cosh r=\cosh s=0, \quad r \neq s .
$$

For the antisymmetric solution (14), an analogous procedure results in

$$
F(\lambda, T)=\sinh r=\sinh s=0, \quad r \neq s .
$$

For $r=s$, using (15) and (16), it can be easily shown that

$$
F(\lambda, T)=r \cosh ^{2} r=0, \quad r=s,
$$

for the symmetric case and

$$
F(\lambda, T)=r \sinh ^{2} r=0, \quad r=s,
$$

for the antisymmetric case.

For clamped edges,

$$
w=w_{, x}=0 \quad \text { at } x= \pm 1
$$

which, in view of (4), yields

$$
X=X^{\prime}=0 \quad \text { at } x= \pm 1 .
$$

Adopting an analogous procedure as for the simply supported edges, one obtains, for the symmetric case

$$
\begin{aligned}
& F(\lambda, T)=r \tanh r-s \tanh s=0, \quad r \neq s, \\
& F(\lambda, T)=\sinh 2 r+2 r=0, \quad r=s,
\end{aligned}
$$

and for the antisymmetric case

$$
\begin{aligned}
& F(\lambda, T)=r \tanh s-s \tanh r=0, \quad r \neq s, \\
& F(\lambda, T)=\sinh 2 r-2 r=0, \quad r=s .
\end{aligned}
$$

The roots of the transcendental equation $F(\lambda, T)=0$ for fixed values of $T$ define the eigenvalues $\lambda_{n}$ of the system, which are in general complex. It can be shown that $-\lambda_{n}$ and $\pm \lambda_{n}^{*}$ are also the eigenvalues of the system. Corresponding to each $\lambda_{n}$, the solution (4) represents an eigensolution of the form

$$
w_{n}(x, y)=X_{n}(x) e^{-\lambda_{n} \cdot v} .
$$

Considering the case of a plate subjected to end loading at $y=0$, the above solution should decay from this end, i.e., the eigenvalues $\lambda_{n}$ should lie in the first and the fourth quadrants of the complex plane. 
4. Plates without in-plane force. In the absence of in-plane force $(T=0)$, the eigenvalue problem takes the form $F(\lambda)=0$. In this case (10) reduces to

$$
p=2 c_{1} \lambda^{2}, \quad q=c_{2} \lambda^{4} .
$$

Thus from (12),

$$
r=i \lambda b_{2}, \quad s=i \lambda b_{1},
$$

where

$$
b_{1}^{2}=c_{1}+\left(c_{1}^{2}-c_{2}\right)^{1 / 2}, \quad b_{2}^{2}=c_{1}-\left(c_{1}^{2}-c_{2}\right)^{1 / 2} .
$$

Noting that $\cosh (i x)=\cos x$ and $\sinh (i x)=i \sin x$ and in view of (33), equations (21), (22), (27), and (29) reduce, for $r \neq s$ (i.e., $b_{1} \neq b_{2}$ ), to

$$
\begin{aligned}
& F(\lambda)=\cos \lambda b_{1}=\cos \lambda b_{2}=0, \\
& F(\lambda)=\sin \lambda b_{1}=\sin \lambda b_{2}=0,
\end{aligned}
$$

for the symmetric and the antisymmetric cases of simply supported plates, respectively, and to

$$
\begin{aligned}
& F(\lambda)=b_{1} \tan \lambda b_{1}-b_{2} \tan \lambda b_{2}=0, \\
& F(\lambda)=b_{1} \tan \lambda b_{2}-b_{2} \tan \lambda b_{1}=0,
\end{aligned}
$$

for the symmetric and the antisymmetric cases of clamped plates, respectively.

From (33) it is seen that for $r=s$ (i.e., $b_{1}=b_{2}$ ), the condition is

$$
c_{1}^{2}-c_{2}=0 \text {, }
$$

or

$$
H^{2}=D_{x} D_{y}, b_{1}=b_{2}=c_{1}^{1 / 2} .
$$

In this case, (23) and (24) reduce to

$$
\begin{aligned}
& F(\lambda)=\cos ^{2} \lambda b_{1}=0, \\
& F(\lambda)=\sin ^{2} \lambda b_{1}=0,
\end{aligned}
$$

for the symmetric and the antisymmetric cases of simply supported plates, respectively, and $(28)$ and $(30)$ reduce to

$$
\begin{aligned}
& F(\lambda)=\sin 2 \lambda b_{1}+2 \lambda b_{1}=0, \\
& F(\lambda)=\sin 2 \lambda b_{1}-2 \lambda b_{1}=0,
\end{aligned}
$$

for the symmetric and the antisymmetric cases of clamped plates, respectively.

For the simply supported plates, the eigenvalues $\lambda_{n}$ are given by the roots of (35) and (36) which are

$$
\lambda_{n}=\left\{\frac{1}{b_{1}}(2 n-1) \frac{\pi}{2}, \frac{1}{b_{2}}(2 n-1) \frac{\pi}{2}\right\}, \quad n=1,2, \ldots,
$$


for the symmetric case and

$$
\lambda_{n}=\left\{0, \frac{1}{b_{1}} n \pi, \frac{1}{b_{2}} n \pi\right\}, \quad n=1,2, \ldots,
$$

for the antisymmetric case. Such solutions are available in the literature.

5. Simply supported plates with in-plane force. It may be noted that the solution to $F(\lambda, T)=0$ in Sec. 3 can be obtained analytically for the simply supported edge condition. Such solutions lend themselves to analytical investigation into the structure of the eigenvalues which is of considerable theoretical importance.

Consider

$$
F(\lambda, T)=\cosh r=\cosh s=0 .
$$

Taking only the first of the above and noting $\cosh r=\cos (i r)$, one obtains

$$
r_{n}= \pm i(2 n-1) \pi / 2, n=1,2, \ldots \text {. }
$$

Upon substituting the above in (12), one obtains

$$
\lambda^{4}+2\left(c_{1} / c_{2}\right) r^{2} \lambda^{2}+\left(1 / c_{2}\right) r^{2}\left(r^{2}-T\right)=0
$$

The roots of this equation are

$$
\begin{aligned}
\lambda^{2} & =-\left(c_{1} / c_{2}\right) r^{2} \pm\left(1 / c_{2}\right)\left\{c_{1}^{2} r^{4}-c_{2} r^{2}\left(r^{2}-T\right)\right\}^{1 / 2}, \\
\lambda & = \pm\left\{-\left(c_{1} / c_{2}\right) r^{2} \pm\left(1 / c_{2}\right)\left(c_{1}^{2} r^{4}-c_{2} r^{2}\left(r^{2}-T\right)\right)^{1 / 2}\right\}^{1 / 2} .
\end{aligned}
$$

The eigenvalues $\lambda$ can be real, imaginary, or complex, for given material parameters, depending upon the magnitude of the in-plane force parameter $T$.

For $\lambda$ to be real, one of the conditions is that $\lambda^{2}$ in (47) must be real, i.e.,

$$
c_{1}^{2} r^{4}-c_{2} r^{2}\left(r^{2}-T\right) \geqslant 0 .
$$

Noting that $r^{2}<0$, the above reduces to

$$
T \leqslant r^{2}\left(1-c_{1}^{2} / c_{2}\right) \text {. }
$$

The other condition for $\lambda$ to be real follows from (48) which is

$$
-\left(c_{1} / c_{2}\right) r^{2} \geqslant\left(1 / c_{2}\right)\left\{c_{1}^{2} r^{4}-c_{2} r^{2}\left(r^{2}-T\right)\right\}^{1 / 2},
$$

which reduces to

$$
T \geqslant r^{2} \text {. }
$$

Combining (49) and (50), the condition for $\lambda$ to be real is

$$
r^{2}<T \leqslant r^{2}\left(1-c_{1}^{2} / c_{2}\right)
$$

For $\lambda$ to be real (one pair) as well as imaginary (other pair), at least one value of $\lambda^{2}$ in (47) must be negative, hence

$$
-\left(c_{1} / c_{2}\right) r^{2} \leqslant\left(1 / c_{2}\right)\left\{c_{1}^{2} r^{4}-c_{2} r^{2}\left(r^{2}-T\right)\right\}^{1 / 2}
$$


which reduces to

$$
T \leqslant r^{2}
$$

For all $\lambda s$ to be complex, the condition from (47) is

$$
c_{1}^{2} r^{4}-c_{2} r^{2}\left(r^{2}-T\right)<0
$$

which reduces to

$$
T>r^{2}\left(1-c_{1}^{2} / c_{2}\right) \text {. }
$$

From the above discussion, it occurs that for given $c_{1}$ and $c_{2}$, the nature of the eigenvalues will be determined by the range (51), (52), or (53) in which $T$ lies. The three ranges are schematically shown in Fig. 2.

For the case $r=s$ in (12), although the above treatment is valid, since the eigenfunction has a different form from the case $r \neq s$, an independent discussion is of interest. From (12) it is seen that for $r=s$, one has

$$
p^{2}-4 q=0 \quad \text { or } \quad \lambda^{2}=\frac{T}{2\left(c_{1} \pm c_{2}^{1 / 2}\right)} .
$$

Again, since $r=(-p / 2)^{1 / 2}$, it follows that

$$
r^{2}=\lambda^{2} c_{2}^{1 / 2}
$$

which can be rewritten as

$$
T=2\left(1 \pm c_{1} / c_{2}^{1 / 2}\right) r^{2}
$$

This indicates that when $T$ takes the values specified by (56), the case $r=s$ occurs. From (55), it is readily seen that since $r^{2}<0, \lambda$ can take only imaginary values.

The above results hold good for the antisymmetric case also except that one has to consider

$$
F(\lambda, T)=\sinh r=\sinh s=0 .
$$

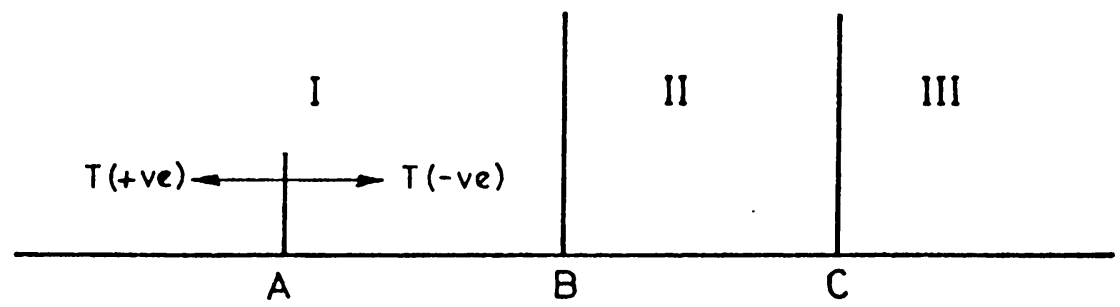

Region I: All $\lambda$ complex (decaying solution with oscillation)

Region II: All $\lambda$ real (decaying solution without oscillation)

Region III: One pair of $\lambda$ real, one pair imaginary (pure oscillation), unstable

A: $T=0 ; \quad$ B: $T=r_{n}^{2}\left(1-c_{1}^{2} / c_{2}\right) ;$ C: $T=r_{n}^{2}$

FIG. 2. Nature of $\lambda_{n}$ in different ranges. 
Taking only the first of the above as before and noting that $\sinh r=-i \sin (i r)$, one obtains

$$
r_{n}= \pm \text { in } \pi, \quad n=1,2, \ldots .
$$

This form of $r_{n}$ must be considered in the previous results.

The illustrative numerical results for typical orthotropic parameters, as will be discussed in Sec. 7, suggested certain behavior of the magnitudes of the eigenvalues $\lambda$ as a function of the in-plane force parameter $T$. This behavior can be predicted analytically and is recorded below.

Firstly, rewrite (53), the range where all $\lambda$ s are complex, as

$$
T>(1-d) r^{2}
$$

or

$$
r^{2}-T<d r^{2}, \quad d=c_{1}^{2} / c_{2},
$$

and (51), the range where all $\lambda \mathrm{s}$ are real, as

$$
r^{2}<T \leqslant(1-d) r^{2}
$$

or

$$
r^{2}-T \geqslant d r^{2},
$$

and note that (52), the range where one pair of $\lambda \mathrm{s}$ is real and the other pair imaginary, can be written as

$$
r^{2}-T \geqslant 0,
$$

thus essentially obeys (59) since $d r^{2}$ is negative. Also rewrite (48), taking only the positive value, as

$$
\lambda=\left[e \pm f\left\{g+h\left(r^{2}-T\right)\right\}^{1 / 2}\right]^{1 / 2}
$$

where

$$
e=-\left(c_{1} / c_{2}\right) r^{2}, \quad f=1 / c_{2}, \quad g=c_{1}^{2} r^{4}, \quad h=-c_{2} r^{2},
$$

and clearly $e, f, g, h$ are all positive. Now considering the value of $\lambda$ at the point

$$
r^{2}-T=d r^{2}
$$

one has

$$
\bar{\lambda}=\left\{e \pm f\left(g+h d r^{2}\right)^{1 / 2}\right\}^{1 / 2},
$$

where the roots $\bar{\lambda}$ are repeated and real by virtue of the equality in (59). Then by (59), it is evident that the multiplicand of $f$ in (61a) is greater than that in (62) and therefore it follows that

$$
\lambda^{(1)}<\bar{\lambda}^{(1)}, \quad \lambda^{(2)}>\bar{\lambda}^{(2)}
$$

where

$$
\lambda^{(1)}<\lambda^{(2)}, \quad \bar{\lambda}^{(1)}<\bar{\lambda}^{(2)} .
$$


Hence, it can be concluded that (i) the lower root $\left(\lambda^{(1)}\right)$ decreases monotonically from the point (62) in the real range (i.e., with decreasing $T$ ) and (ii) the higher root $\left(\lambda^{(2)}\right)$ increases monotonically from the point (62) in the real range (i.e., with decreasing $T$ ). Again it is easy to see that the limiting value of the lower root $\left(\lambda^{(1)}\right)$ is zero and $\lambda^{(2)}$ continues to increase monotonically with decreasing $T$ in the range (60).

Turning to the complex range (58), one has

$$
g+h\left(r^{2}-T\right)<0, \quad r^{2}-T<0,
$$

and thus the imaginary part of $\lambda^{2}$ is given by

$$
\operatorname{Im}\left\{\lambda^{2}\right\}=f\left\{g+h\left(r^{2}-T\right)\right\}^{1 / 2} .
$$

Clearly by (58) and (61a)

$$
\operatorname{Im}\left\{\lambda^{2}\right\}>\operatorname{Im}\left\{\bar{\lambda}^{2}\right\}, \quad \operatorname{Re}\left\{\lambda^{2}\right\}=\operatorname{Re}\left\{\bar{\lambda}^{2}\right\} .
$$

Thus,

$$
\because \operatorname{Re}\{\lambda\}>\operatorname{Re}\{\bar{\lambda}\}, \quad \operatorname{Im}\{\lambda\}>\operatorname{Im}\{\bar{\lambda}\} .
$$

Hence, it can be concluded that the real as well as the imaginary part of $\lambda$ increases monotonically from the point (62) in the complex range (i.e., with increasing $T$ ). This fact, however, is obvious from physical reasoning as well.

The case of isotropic plate. For the isotropic plate the foregoing results can be readily specialized by noting that $c_{1}=c_{2}=1$ and the definitions of $r$ and $s$ in (12) remain unaltered with

$$
p=2 \lambda^{2}-T, \quad q=\lambda^{4} .
$$

The ranges (51), (52), and (53) for the values of $T$ reduce, respectively, to

$$
r^{2}<T \leqslant 0, \quad T<r^{2}, \quad T>0 .
$$

For $r=s,(54)$ and (56) reduce, respectively, to

$$
\lambda^{2}=T / 4, \quad T=4 r^{2},
$$

and as before $\lambda$ can take only imaginary values.

6. Behavior of eigenvalues of simply supported plates. When the eigenvalues (roots of (46)) are all real, given by the range in (51), from the physical point of view, the end loading will decay (from $y=0$ ) depending upon the magnitude of the positive pair, and the negative pair can be ignored. This can be taken to be a stable solution (see region II in Fig. 2).

When the eigenvalues are all complex, given by the range in (53), from the physical point of view, the end loading displays an oscillatory decay (from $y=0$ ). The pair with negative real parts can be ignored and the solution again is stable (see region I in Fig. 2).

When one pair of eigenvalues is real and the other pair imaginary, the negative real root as well as the negative imaginary root can be ignored. The nature of such a solution implies an unstable situation because the end loading has an oscillatory component without decay (from $y=0$ ) corresponding to the nonzero imaginary eigenvalue (see 
region III in Fig. 2). In the special case of $T=r^{2}$, which is a critical point, it follows from (46) that the four eigenvalues are

$$
\lambda=\left\{0,0, \pm(2 n-1) \frac{\pi}{\sqrt{2}}\left(c_{1} / c_{2}\right)^{1 / 2}\right\}, \quad n=1,2, \ldots,
$$

for the symmetric mode, and

$$
\lambda=\left\{0,0, \pm n \pi \sqrt{2}\left(c_{1} / c_{2}\right)^{1 / 2}\right\}, \quad n=1,2, \ldots,
$$

for the antisymmetric mode. In view of the zero roots above, the plate cannot sustain an end loading, in other words, the bending is transmitted without decay from $y=0$ to $y=\infty$. The critical values of $T$ may be written as

$$
T_{m}=-m^{2} \pi^{2} / 4, \quad m=1,2, \ldots,
$$

where the odd $m$ represents the symmetric modes and the even $m$ represents the antisymmetric modes. The above equation may be rewritten in view of (7) as

$$
\bar{N}_{m}=-m^{2} \pi^{2} D_{x} / 4 a^{2}
$$

where $\bar{N}_{m}$ are the critical values, which are the well-known buckling values of a semi-infinite plate with only $N_{x}$ acting. The index $m$ represents the number of half waves of the buckled mode of the plate in the $x$-direction. The in-plane load $N_{x}$ can be conveniently written as

$$
N_{x}=\beta N_{c r}=\beta \bar{N}_{1},
$$

where $N_{c r}$ is the first buckling load of the plate.

It is of interest to view the above analysis in conjunction with the dynamic formulation of linear bifurcation (buckling) problem. In the latter case, the frequencies of vibration turn out to be zero at the critical points, and hence this condition is taken as the dynamic criterion of buckling. In the same manner, for the semi-infinite orthotropic plate problem studied here, the critical points, corresponding to (71), denote zero eigenvalues which are associated with the decay of bending due to end loading. Thus, this approach could be used to provide an alternate criterion for the classical buckling problem, and it is of interest to explore further the application of this approach to more general situations. The present formulation evidently integrates the eigenvalue problems of bending and buckling leading to a combined eigenvalue problem which can be written in general terms as

$$
G(\lambda)=F\left(\lambda_{n}^{(j)}, r_{n}, T_{m}\right)=0, \quad j=1,2,3,4 .
$$

As discussed in Sec. 5, the nature of eigenvalues depends upon the value of the in-plane load parameter $T$. When $T=T_{m}$, the critical condition arises. When $T$ lies between any two successive critical loads, i.e.,

$$
T_{m}^{2}<T<T_{m-1}^{2},
$$

it can be easily shown that, for all $n<m$, of the four values of $\lambda_{n}$, one pair will be imaginary and the other pair will be real, thus signifying an unstable situation. For all $n \geqslant m$, the $\lambda_{n}$ may be all real or all complex depending upon the material parameters, which signifies a stable situation. 
7. Numerical illustrations. In order to illustrate the effect of the material parameters $\left(c_{1}, c_{2}\right)$ on the eigenvalues, the case of simply supported plates with $T=0$ has been considered. The plots of $\lambda_{n}\left(n=1\right.$ to 5) against $c_{1}\left(=c_{2}\right)$ are presented in Figs. 3 and 4. The range of values of $c_{1}$ is very wide where the lower values represent composite laminates etc. and the higher values represent stiffened steel panels used in ship and bridge structures. The eigenvalues for the clamped plate for a typical stiffened panel are presented in Table 1. In order to illustrate the effect of the in-plane force parameter $(T)$ on the eigenvalues of the simply supported case, three different values of $c_{1}\left(=c_{2}\right)$ have been considered. The plots of $\lambda_{1}$ and $\lambda_{2}$ against $\beta$ are presented in Fig. 5.

It is observed from Figs. 3 and 4 that $\operatorname{Re}\left\{\lambda_{n}\right\}>\operatorname{Im}\left\{\lambda_{n}\right\}$ (but only slightly) and $\lambda_{n}$ lies on a straight line in the complex plane. The effect of edge conditions on $\lambda_{n}$ is obvious from Table 1.

As indicated in (74), the eigenvalues occur in clusters of 4 for every $n$. Fig. 5 brings out graphically the nature of roots in various ranges as discussed in Sec. 5. In general it is seen that the lower value of $\lambda_{1}$ increases with increasing $T$, the increase being more pronounced for smaller $c_{1}$. In contrast, the corresponding increase in $\lambda_{2}$ with $T$ is significantly less.

In Fig. 5 only the effect of $\beta$ on $\lambda_{1}$ up to $\beta=-1\left(T=T_{1}\right)$ could be shown. A qualitative picture of the behavior of higher eigenvalues $\left(\lambda_{2}\right.$ and $\left.\lambda_{3}\right)$ versus $\beta$ covering the ranges $T_{2}$ and $T_{3}$ also is shown in Fig. 6. The unstable regions of the solution corresponding to $\lambda_{1}, \lambda_{2}$, and $\lambda_{3}$ are as indicated.

8. Conclusion. The eigenfunction formulation of a semi-infinite orthotropic plate with in-plane force in the finite direction has been studied with particular reference to simply supported edge condition. The eigenspectrum consists of clusters of values, with four in

TABLE 1. Eigenvalues for typical stiffened plate parameters

\begin{tabular}{|c|c|}
\hline Simple Support & \multicolumn{1}{|c|}{ Clamped Support } \\
\hline $4.977171+i 4.722799$ & $7.4094152+i 7.198859$ \\
$14.93152+i 14.1684$ & $17.34127+i 16.612993$ \\
$24.88586+i 23.61399$ & $27.295599+i 26.058526$ \\
$34.8402+i 33.0596$ & $37.24995+i 35.504131$ \\
$44.79454+i 42.50518$ & $47.204299+i 44.949737$ \\
\hline
\end{tabular}

$$
\begin{aligned}
& \text { Panel Data } \\
& \text { Young's Modulus }=2.1 \times 10^{6} \mathrm{~kg} . \mathrm{cm}^{-2} \\
& \text { Poisson's ratio }=0.3 \\
& D_{x}=84.3972 \times 10^{6} \mathrm{~kg} . \mathrm{cm}, \\
& D_{y}=0.23184 \times 10^{6} \mathrm{~kg} . \mathrm{cm}, \\
& D_{1}=0.069552 \times 10^{6} \mathrm{~kg} . \mathrm{cm} \\
& D_{x y}=0.081144 \times 10^{6} \mathrm{~kg} . \mathrm{cm}, \\
& c_{1}=c_{2}=0.00274701 .
\end{aligned}
$$




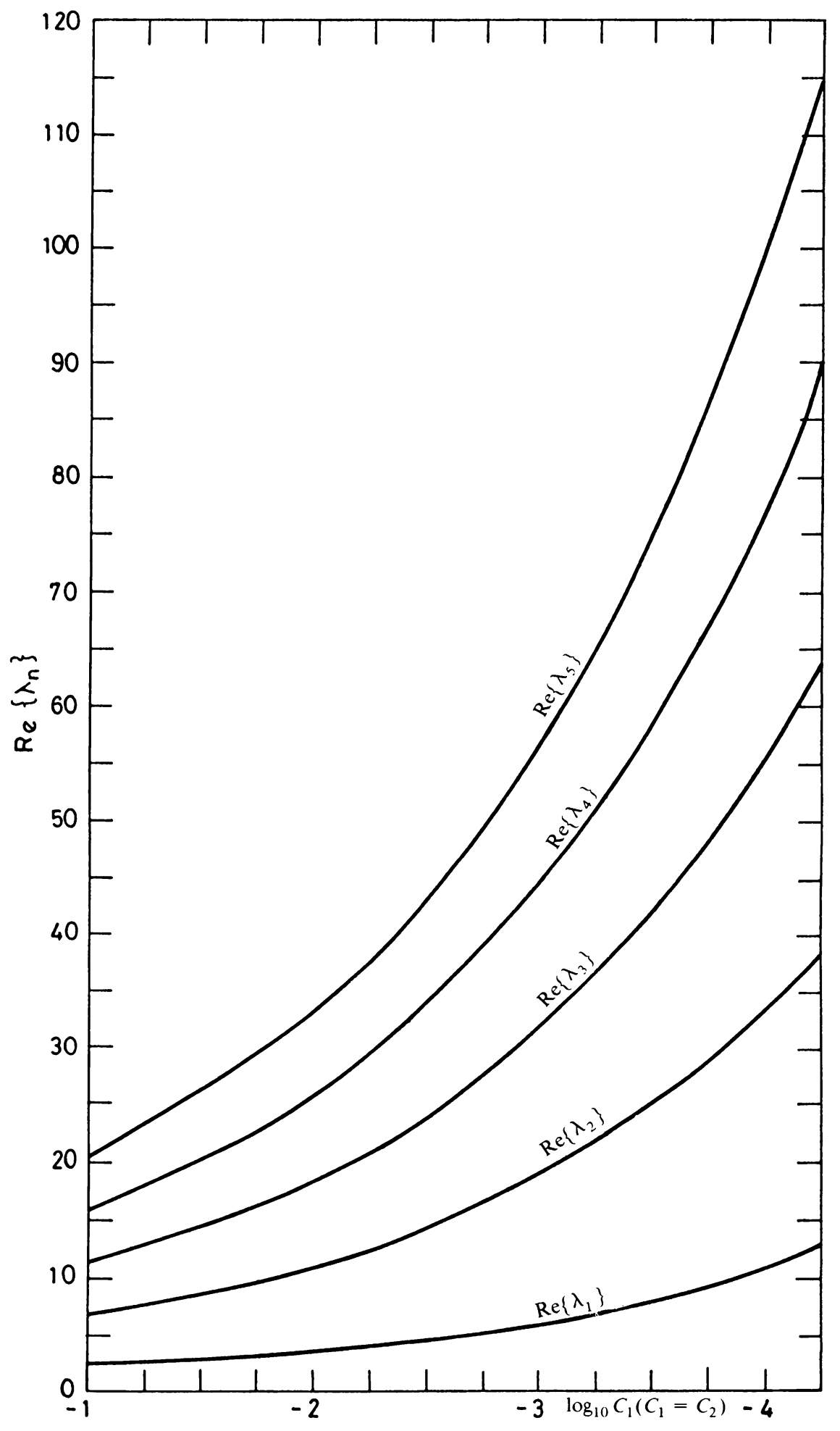

FIG. 3. $\operatorname{Re}\left\{\lambda_{n}\right\}$ at $T=0$. 


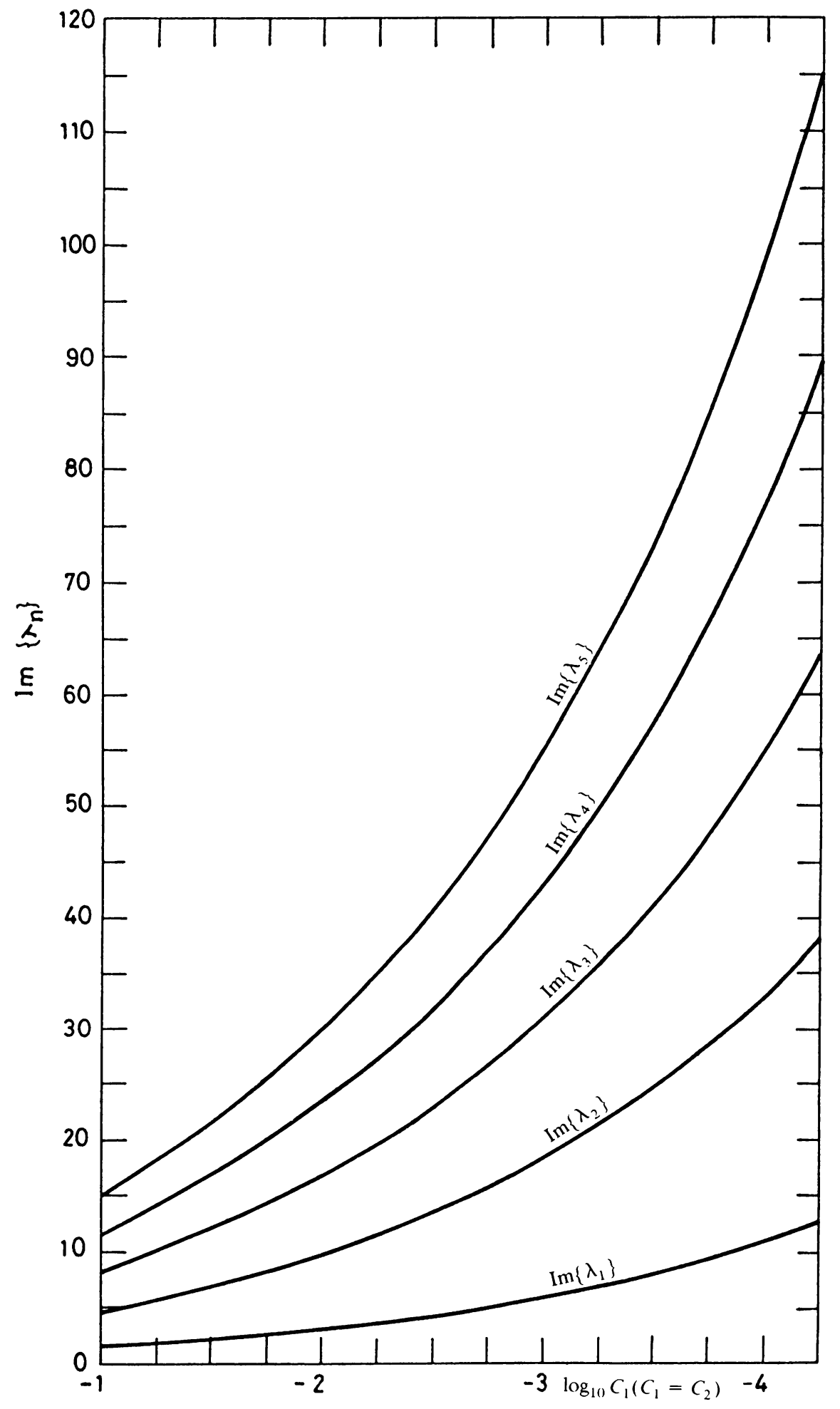

FIG. 4. $\operatorname{Im}\left\{\lambda_{n}\right\}$ at $T=0$ 


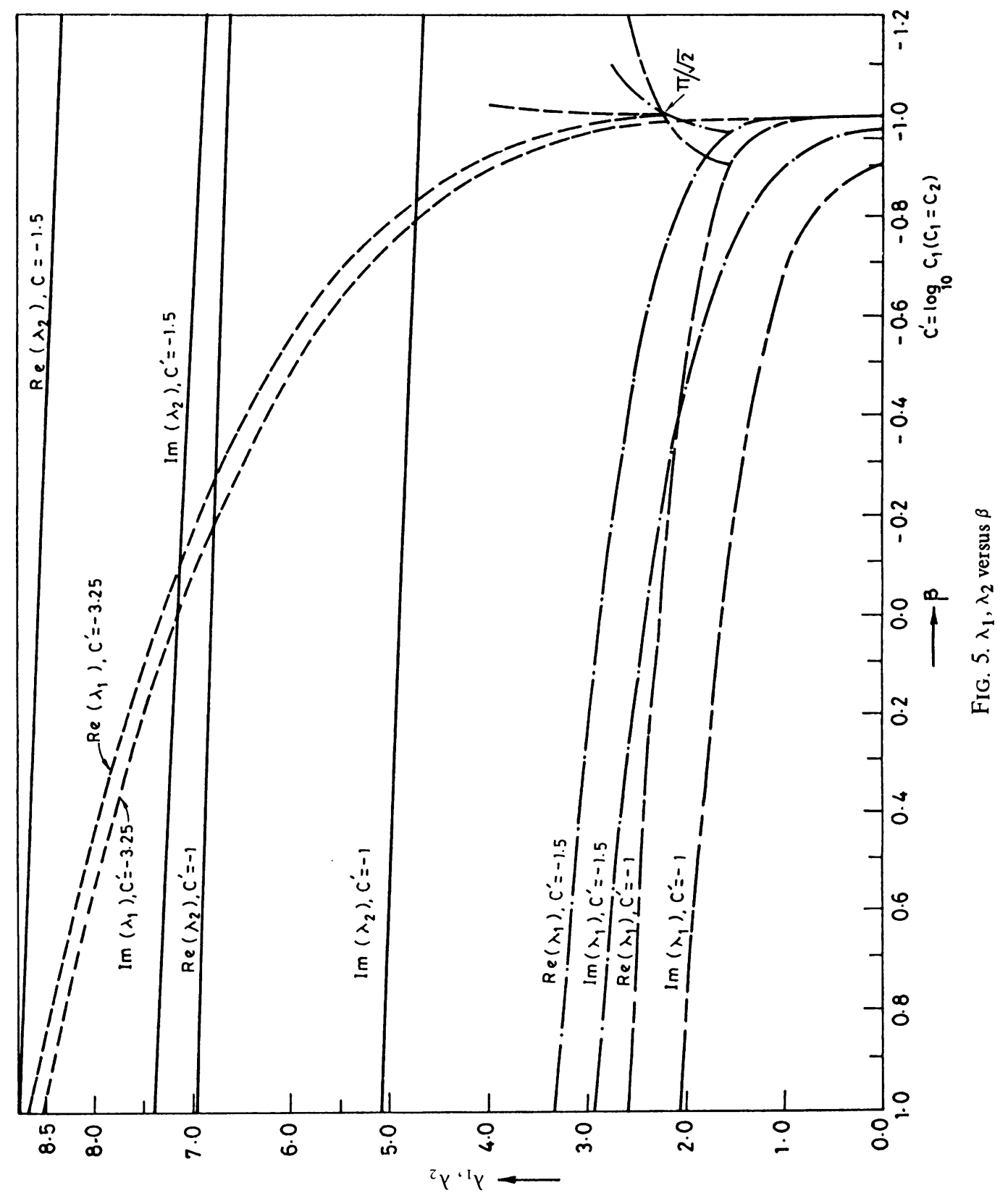




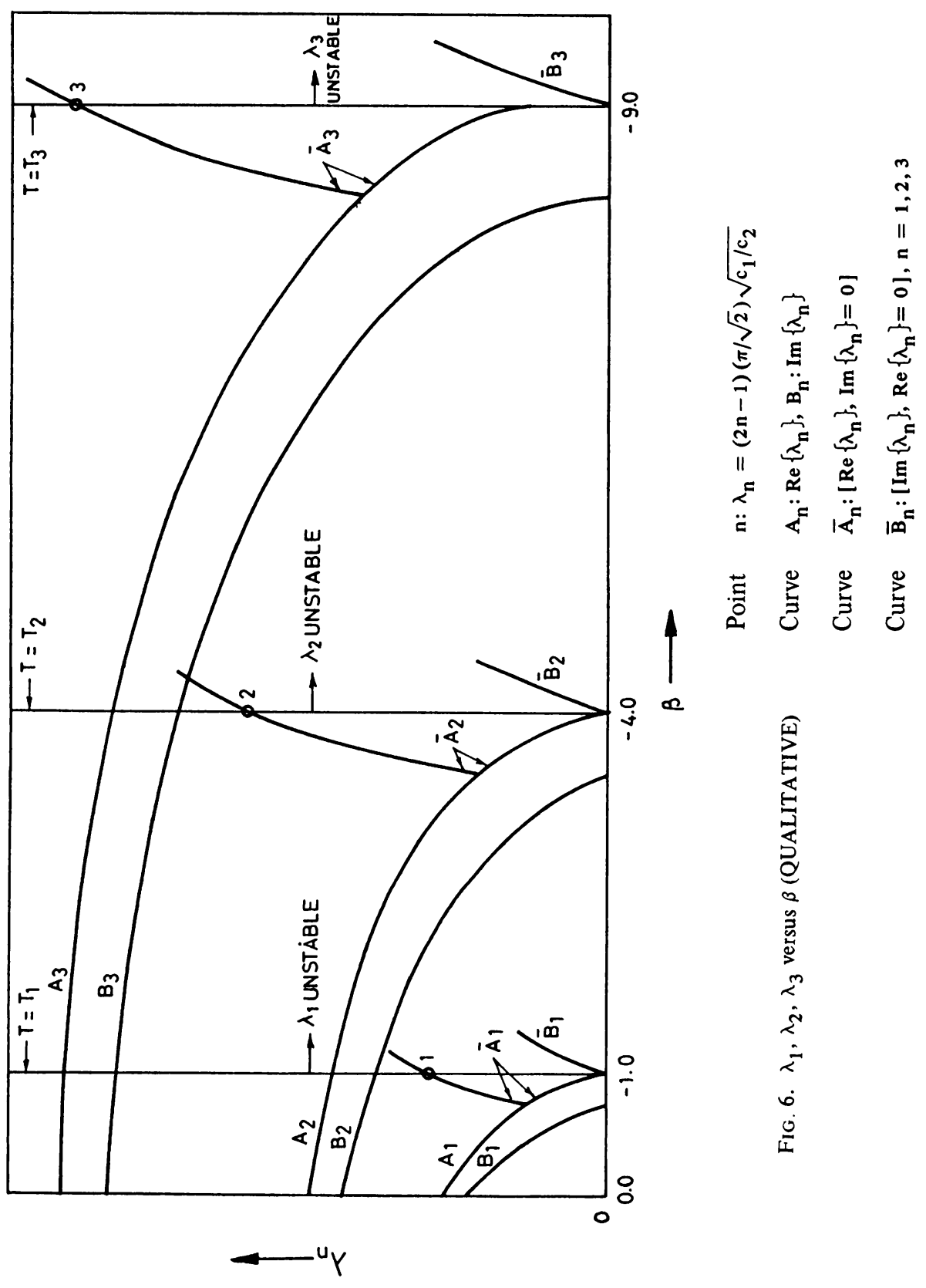


each cluster. The eigensolutions essentially represent the decay of bending corresponding to end loading. The magnitudes of the eigenvalues $\lambda_{n}$, especially $\lambda_{1}$, indicate the strength of decay. The characteristics of the eigenvalues in different ranges of $T$ have been brought out analytically for the simply supported edge condition. The treatment of the case of clamped plates must be done numerically. However, the characteristics of the eigenvalues will essentially be the same as in the simply supported case. It is seen that the present formulation can identify certain regions of static instability on the basis of the nature of the eigenvalues. Thus it integrates the eigenvalue problem of bending and that of linear bifurcation buckling. The occurrence of eigenvalues indicating nondecaying solutions denotes instability as discussed in the present paper which suggests an alternate criterion for linear bifurcation buckling. In this context, it is of interest to recall the well-known dynamic criterion of bifurcation instability. A more general investigation of this problem will be of considerable interest.

\section{REFERENCES}

[1] J. Fadle, Die Selhstspannungs-Eigenwert funktionen der quadratischen Scheibe, Ingen. Archiv. 11, $125-149$ (1940)

[2] P. F. Papkovitsch, Üher eine Form der Lösung des byharmonischen Problems für das Rechteck. C. R. Doklady, Academy of Science, URSS (N.S.) 27, 334-338 (1940)

[3] P. F. Papkovitsch, Zwei Fragen zur Theorie der dünnen elastischen Platten. J. Appl. Math. Mech. [Akad. Nauk SSSR. Zhurnal Prikl. Mat. Mech.] 5, 359-374 (1941)

[4] J. L. Klemm and R. W. Little, Saint-Venant's principle, Tech. Report No. 10, Div. of Engg. Research, Michigan State Univ. (1971)

[5] L. S. D. Morley, Simple series solution for the bending of a clamped rectangular plate under uniform normal load, Quart. J. Mech. \& Appl. Math. (1963)

[6] F. A. Gaydon, The clamped, thin, rectangular plate under transverse loading, Quart. J. Mech. \& Appl. Math. (1966)

[7] B. S. Ramachandra Rao, G. L. Narasimham and S. Gopalacharyulu, Eigenfunction analysis for bending of clamped, rectangular, orthotropic plates, Int. J. Solids and Structures (1973)

[8] R. R. Archer and N. Bandopadhyay, On the 'end problem' for thick rectangular plates, Mechanics Today, vol. 5, Ed. by S. Nemat-Nasser, pp. 1-13, Pergamon, Oxford, (1978)

[9] M. S. Troitsky, Stiffened plates-bending, stability and vibrations, Elsevier, 1976 